\title{
Metformin, cancer and glucose metabolism
}

\author{
Barbara Salani ${ }^{1,2}$, Alberto Del Rio ${ }^{3,4}$, Cecilia Marini ${ }^{5}$, Gianmario Sambuceti ${ }^{2,6}$, \\ Renzo Cordera ${ }^{1,2}$ and Davide Maggi ${ }^{1,2}$ \\ ${ }^{1}$ Department of Internal Medicine (DIMI), University of Genova, Viale Benedetto XV/6, 16132 Genova, Italy \\ ${ }^{2}$ IRCCS Azienda Ospedaliera Universitaria San Martino - IST Istituto Nazionale per la Ricerca sul Cancro, \\ 16132 Genova, Italy \\ ${ }^{3}$ Department of Experimental, Diagnostic and Specialty Medicine (DIMES), Alma Mater Studiorum, \\ University of Bologna, Bologna, Italy \\ ${ }^{4}$ CNR Institute of Organic Synthesis and Photoreactivity (ISOF), 40129 Bologna, Italy \\ ${ }^{5} \mathrm{CNR}$ Institute of Molecular Bioimaging and Physiology (IBFM), 16132 Genova, Italy \\ ${ }^{6}$ Department of Health Science (DISSAL), University of Genova, 16132 Genova, Italy
}

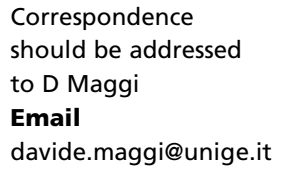

\begin{abstract}
Metformin is the first-line treatment for type 2 diabetes. Results from several clinical studies have indicated that type 2 diabetic patients treated with metformin might have a lower cancer risk. One of the primary metabolic changes observed in malignant cell transformation is an increased catabolic glucose metabolism. In this context, once it has entered the cell through organic cation transporters, metformin decreases mitochondrial respiration chain activity and ATP production that, in turn, activates AMP-activated protein kinase, which regulates energy homeostasis. In addition, metformin reduces cellular energy availability and glucose entrapment by inhibiting hexokinase-Il, which catalyses the glucose phosphorylation reaction. In this review, we discuss recent findings on molecular mechanisms that sustain the anticancer effect of metformin through regulation of glucose metabolism. In particular, we have focused on the emerging action of metformin on glycolysis in normal and cancer cells, with a drug discovery perspective.
\end{abstract}

\section{Key Words \\ - Metformin \\ - Cancer \\ - Glucose \\ - Hexokinase \\ - AMPK}

Endocrine-Related Cancer (2014) 21, R461-R471

\section{Introduction}

Metformin is a biguanide, whose chemical scaffold was discovered by the extraction of the galegine, a guanidine analogue, from French lilac (Galega officinalis) plants. Metformin is the first-line therapy for type 2 diabetes (Alexander et al. 2008). In 2005, a 23\% reduction in the incidence of any cancer in type 2 diabetic patients treated with metformin was reported (Evans et al. 2005). In recent years, results from retrospective epidemiological and in vitro studies have supported the rationale of designing clinical trials using metformin as an adjuvant in chemotherapy for cancer patients (Zhang et al. 2013). Conversely, the recently raised criticisms about the role of metformin in cancer have underlined the clinical heterogeneity between trials and the presence of timerelated biases (Stevens et al. 2012, Suissa \& Azoulay 2012, Badrick \& Renehan 2014). Indeed, recent experimental results have shed new light on the mechanisms of action of metformin as a key regulator of cellular metabolism. It has been reported that metformin inhibits proliferation and induces apoptosis in cancer cells as a result of decreased energy disposition due to an increased AMP:ATP ratio and AMP-activated protein kinase (AMPK) activation (Viollet et al. 2011). The ability of metformin to mimic a condition of caloric restriction is currently of great interest in the

Published by Bioscientifica Ltd 
field of oncology (Omar et al. 2010). In fact, to compensate the rapid cell growth and proliferation, cancer cells exploit all possible mechanisms, including increased metabolism, demand for nutrients and consumption of glucose, which is known as the Warburg effect (Koppenol et al. 2011). Results of recent studies have indicated that metformin treatment might inhibit glucose uptake in tumours. In this review, we describe findings supporting the effect of metformin on glucose metabolism in normal and cancer cells.

\section{Metformin and organic cation transporters in diabetes and cancer}

In diabetic patients, metformin decreases plasma glucose concentration mainly by decreasing hepatic gluconeogenesis and glucose output (Wollen \& Bailey 1988). This effect is followed by an increase in glucose uptake (Iozzo et al. 2003) and the amelioration of insulin resistance. Metformin treatment, through AMPK activation, increases insulin-activated but not basal glucose uptake in muscle (Hundal et al. 1992, Sajan et al. 2010, Kristensen et al. 2014). In all cases, the potentiation of glucose uptake in insulin target tissues was mostly observed after long-term treatment with metformin and the effect was associated with restoration of insulin sensitivity (Natali \& Ferrannini 2006). Owing to its physicochemical properties, metformin is not transported across biological membranes by passive diffusion but mainly by organic cation transporters (OCTs), which are therefore determinants of metformin disposition and therapeutic efficacy. The fact that the main responsive tissue to metformin is the liver is supported by the expression pattern of the principal metformin transporter, the solute carrier family 22 member 1 (SLC22A1), also known as OCT1, which is almost exclusively expressed in the liver (Zhang et al. 1997). Two other OCT isoforms have been identified. Cellular intake of metformin can be mediated also by OCT2 (gene SLC22A2) and OCT3 (gene $S L C 22 A 3)$. OCT2 is absent in the liver and highly expressed in renal tubules where it mediates the uptake of metformin from the circulation into renal epithelial cells. Compared with OCT1 and OCT2, ОСТ3 shows a much broader tissue distribution and it has been proposed to be a new transporter of metformin in skeletal muscle, heart, brain, placenta and liver (Aoki et al. 2008, Chen et al. 2010). Excluding adrenal gland, OCT3 is weakly expressed in other tissues including liver where the OCT1:OCT3 mRNA ratio reaches 34 (Nies et al. 2009). Hepatic uptake of metformin is primarily mediated by OCT1. Liver accumulates the highest amount of metformin via the portal circulation and the high level of expression of OCT1. At the same time, OCT2 plays an important role in the disposition of metformin because renal secretion is the major elimination route of metformin. Metformin in fact is not metabolised but excreted unchanged in the urine with a half-life of about $6 \mathrm{~h}$ (Graham et al. 2011). It is important to note that OCT1 and OCT2 expression levels as well as their genetic polymorphisms contribute to the inter-individual variability in response to metformin treatment (Shu et al. 2008, Takane et al. 2008). Indeed, it has been shown that several genetic variations in OCT1 and OCT2 are associated respectively with a reduced metformin uptake or increased elimination causing significant changes in plasma concentrations of metformin and hypoglycaemic effects (Choi \& Song 2008). Combined impairment of OCT1 and OCT2 in doubleknockout mice decreases hepatic metformin uptake to 76\% (Higgins et al. 2012). Compensatory mechanisms such as passive diffusion seem unlikely because of the polarity and low membrane permeability of metformin. While the virtual absence of OCT1 expression is associated with noncancerous tissues, tumour cells significantly express OCT1 and its downregulation limits the antineoplastic effect of metformin (Segal et al. 2011, Gupta et al. 2012). In this scenario, metformin's ability to increase OCT1 expression may represent an important tool for potential applications in oncology (Salani et al. 2012). For instance, pyriplatin, which is a member of the platinum analogues and has never been tested in humans, provides an example of a drug distinct from metformin, for which OCT1 may play a role in its pharmacokinetic and toxicity. Using OCT1 knockout mice, it has been demonstrated that OCT1 expression facilitates the accumulation of pyriplatin (Li et al. 2011). Also, tyrosine kinase inhibitors such as imatinib, used in the treatment of chronic myeloid leukemia, are transported by OCT1 (Eechoute et al. 2011), though the relationship between OCT1 genetic profile and drug responsiveness is still debated (Grinfeld et al. 2013, Koren-Michowitz et al. 2013, Nies et al. 2014). Further studies on genetic variants of OCT1 could help to predict the efficacy of both metformin and platinum-based drugs (Zhang et al. 2006).

\section{Metformin and glucose cell metabolism}

\section{Canonical effects of metformin on glucose metabolism}

One of the most discussed therapeutic actions of metformin in diabetes is the inhibition of gluconeogenesis and hepatic glucose output obtained by the disruption of

Published by Bioscientifica Ltd. 
energy metabolism, mainly characterised by an increase in the AMP:ATP ratio (El-Mir et al. 2000). These results indicate that AMPK, a serine/threonine kinase that regulates cellular homeostasis in response to energy stress and energy unbalance, is the primary target of metformin (Viollet et al. 2011). AMPK is a hetero-trimetric protein composed of one catalytic $(\alpha)$ and two regulatory subunits ( $\beta$ and $\gamma$ ). When intracellular ATP concentration decreases, AMP or ADP binds the $\gamma$ regulatory subunit leading to a conformational change that protect against phosphorylation of AMPK in Thr172 by the serine/threonine kinase LKB1 (Hawley et al. 2003) or in response to calcium flux via CAMKK2 kinase (Hawley et al. 2005). Metformin treatment and caloric restriction increase the AMP:ATP ratio and activate AMPK, switching cells from an anabolic to a catabolic state. In particular, through AMPK pathway, metformin induces the phosphorylation of CREB-regulated transcription co-activator 2 (CRTC2 or TORC2), sequestering CRTC2 from the nucleus to the cytoplasm (Shaw et al. 2005). This mechanism inhibits the transcriptional activation of gluconeogenic genes such as phosphoenolpyruvate (PEP) carboxykinase (PEPCK) (PCK2) and glucose-6-phosphatase (G6Pase). Whether AMPK is absolutely required to decrease circulating plasma glucose concentration remains controversial. Metformin, in fact, can also decrease hepatic gluconeogenesis independently of the LKB1/AMPK pathway as demonstrated in AMPK- and LKB1-deficient hepatocytes (Foretz et al. 2010). The inhibition of liver gluconeogenesis in these models seems related to energy state changes associated with mitochondrial function and does not involve gluconeogenic gene expression processes.

The multiplicity of effects of metformin also includes the reduction in gluconeogenesis in liver cells due to the decreased uptake of gluconeogenic substrates such as L-alanine (Komori et al. 1993) and lactate (Radziuk et al. 1997) and the direct inhibition of the redox shuttle enzyme glycerophosphate dehydrogenase (Madiraju et al. 2014). Metformin also reduces glucose output through the decrease in cAMP, protein kinase A activity and phosphorylation of protein kinase A substrates induced by glucagon (Miller et al. 2013). The stimulatory effect of metformin on glycogenolysis and glycolysis is the consequence of reduced oxidative phosphorylation coupled with the inhibition of oxygen consumption. These effects do not necessarily require AMPK activation because changes in AMP:ATP ratio can allosterically influence the enzymatic activity of several enzymes of the glycolytic pathway. High levels of ATP, for instance, allosterically inhibit phosphofructokinase and pyruvate kinase, which are important control elements in glycolysis.

Respiratory chain inhibition has been identified as the first mechanism by which metformin induces significant changes in total cellular ATP content (El-Mir et al. 2000). Observing the decreased oxygen consumption and mitochondrial membrane potential in permeabilised cells and isolated mitochondria, the existence of a direct effect on complex 1 of the respiratory chain was proposed (Owen et al. 2000). This effect could be the result of molecular changes occurring in the inner mitochondrial membrane due to the binding of metformin to phospholipids (Drahota et al. 2014). Modification of membrane permeability and fluidity may induce changes in mitochondrial proteins and functionality by interfering with the $V_{\max }$ of catalysis and the conformations of important mitochondrial proteins, including respiratory chain complex 1. These indirect mechanisms could promote mitochondrial injury and result in a predisposition to cell death.

\section{Effect of metformin on glucose metabolism in cancer}

The first identified cellular target of metformin linking AMPK and cancer came from the discovery of $L K B 1$, a tumour suppressor gene, which was identified as the major upstream activator of AMPK (Shaw et al. 2004). The LKB1-AMPK interaction mediates glucose homeostasis in liver and play an important role in the suppression of protein synthesis, a process often deregulated in cancer (Zakikhani et al. 2006). Activation of LKB1-AMPK induced by metformin results in the inhibition of Raptor-mTOR (mammalian TOR complex 1(mTORC1)) complex and suppression of cellular protein synthesis and growth (Dowling et al. 2007). Phosphorylation of AMPK activates tuberous sclerosis complex 2 (TSC2), a subunit of the TSC1-TSC2 (hamartin/tuberin) complex. The TSC1-TSC2 complex, through its GTPase-activating protein, inactivates the small GTP-binding protein Ras Homolog Enriched in Brain (RHEB). Once bound to GDP, RHEB cannot activate mTORC1, which decreases mRNA translation and ribosome biogenesis affecting the cell cycle and proliferation (Inoki et al. 2006). Metformin can exert its anti-neoplastic effect by inhibiting pathways downstream of AMPK without any activation of AMPK, as demonstrated in lung cancer (Memmott et al. 2010). AMPKindependent effects of metformin in cancer, such as the inhibitory effect on AKT phosphorylation in breast and lung cancers, has been well documented and associated also with decreased plasma concentrations of insulin-like

Published by Bioscientifica Ltd. 
growth factor 1 (IGF1) and insulin (Pollak 2012). Thus, the actions of metformin are not exclusively mediated by inhibition of AMPK (Watson 2014).

Indeed, in recent years new effects of metformin upstream of AMPK activation have been identified. In particular, metabolic changes have been rediscovered in the context of cancer diseases after the initial observations of Otto Warburg, who noticed that proliferating cancer cells consume glucose at a high rate, releasing lactate and not carbon dioxide (Koppenol et al. 2011). The tumour cells avidly use glucose as a source of energy and for production of cell-building blocks. Critical to this phenotype is the production of glucose-6-phosphate (G6P), whose role in glucose retention and metabolism is highly advantageous for cancer cell survival and proliferation (Vander Heiden et al. 2009). Glucose phosphorylation is the first step in glucose metabolism. This reaction is catalysed by hexokinases (HKs), enzymes that exist in four isozymic forms, differing in catalytic and regulatory properties as well as subcellular localisation. HK1, -2 and 3 are $100 \mathrm{kDa}$ molecules that show a high affinity for glucose, as demonstrated by their $\mathrm{Km}$ values in the micromolar range, and are very sensitive to the inhibition by their product G6P. The type 1 isozyme is ubiquitously expressed, while the type 2 is found in skeletal muscle, adipose tissue and heart; the type 3 is limited to neurons. In contrast, HK4, also referred as glucokinase, is expressed in liver and pancreas. HK4 has a molecular mass of $50 \mathrm{kDa}$, exhibits a lower affinity for glucose and represents the only HK that is not inhibited by G6P (Wilson 2003). HKs 1 and 2 have been found in mitochondrial fraction of several tissues (Wilson 2003). Binding of HKs 1 and 2 to mitochondria represents a privileged access to ATP produced by the respiratory chain. In the outer mitochondrial membrane, in fact, HK1 and 2 interact with the permeability transition pore which includes the voltagedependent anion channel 1 (VDAC1) responsible for ATP flux to the cytoplasm. This physical association facilitates glucose phosphorylation and protects cell from apoptosis (Pastorino \& Hoek 2003). HK2 is highly expressed in lung and breast cancer and is required by proliferating cancer cells as demonstrated by the impairment of tumour progression following its downregulation (Fang et al. 2012, Patra et al. 2013). In the unbound state, HK2 exists in an open inactive structure (Fig. 1a), where ATP is bound within the large lobe but in a conformation far from the catalytic site. Upon glucose binding, a large conformational change closes the two lobes resulting in an enzymatically active closed HK2 that presents ATP in a productive conformation protruding towards the sugar substrate (Fig. 1). Metformin directly inhibits HK2 activity and subcellular localisation inducing dissociation of HK2 from the mitochondria (Fig. 2). The region occupied by metformin has been simulated by computational modelling and found to correspond with the binding site of G6P. Accordingly, it was found that metformin does not inhibit glucokinase

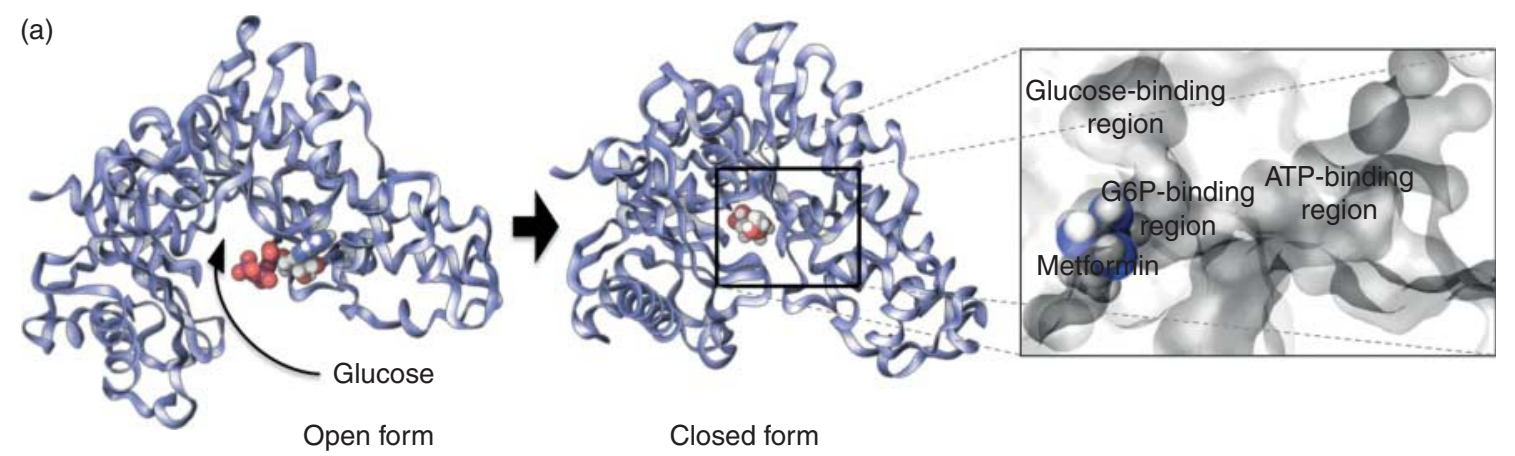

(b)
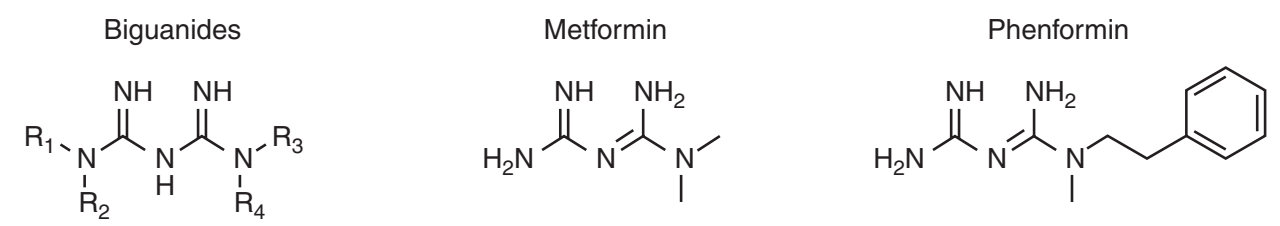

\section{Figure 1}

(a) Crystallographic structure of HK2 in open (based on PDB ID: 1IG8) and closed (based on PDB ID: 2NZT) conformations in complex with glucose and ATP and a close-up picture of the catalytic site with the putative binding mode of metformin in the G6P binding region. (b) Chemical structure of biguanide analogues, metformin and phenformin. 
HK4, which is the only HK isoform that does not show any auto-inhibition by its product G6P (Salani et al. 2013). Glycolysis and mitochondrial oxidative phosphorylation are targets of biguanides.
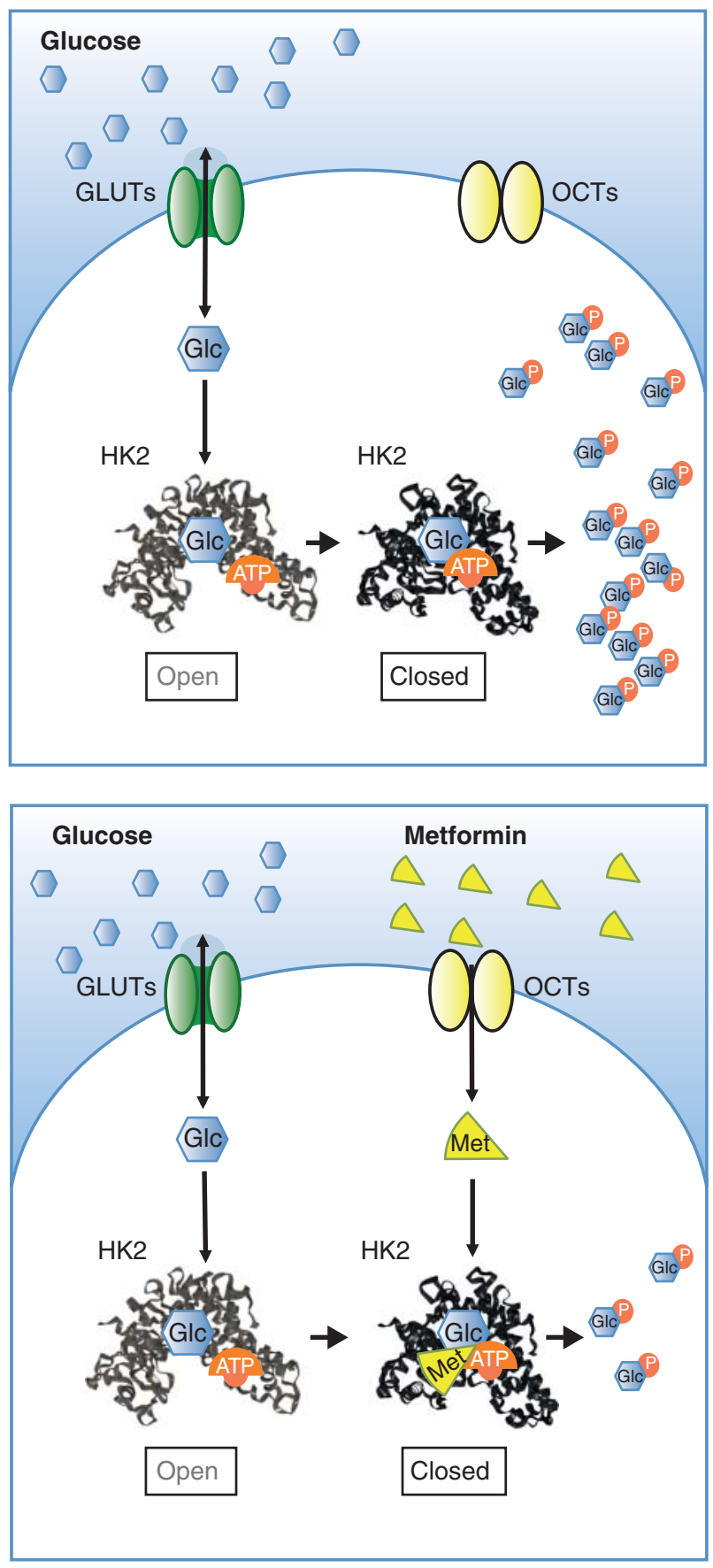

Figure 2

Metformin's inhibitory effect on HK2 activity. Binding of glucose to HK2 is required to induce conformational change in the enzyme from an open to a closed structure. Metformin inhibits HK2 only in the closed conformation. The region occupied by metformin corresponds to the binding site of G6P, an inhibitor of the enzymatic reaction.
Recent findings have shown that these compounds act more effectively in cellular models with defects in glucose utilization and mitochondrial function. For instance, mitochondrial DNA mutations in complex 1 genes are the determinants of cancer cell's sensitivity to phenformin when cells are grown with low glucose levels (Birsoy et al. 2014). Vice versa, high glucose concentrations reduce the effectiveness of metformin on breast cancer cells' proliferation (Wahdan-Alaswad et al. 2013). All these findings indicate that the interaction between glucose dose and metformin efficacy could have implications for the clinical use of metformin (Wahdan-Alaswad et al. 2013, Zordoky et al. 2014). In particular, the maintenance of glucose homeostasis could represent an important requirement for the clinical use of metformin for oncological treatments.

\section{Insulin action and metformin: a metabolic point of view}

Although several studies have shown that metformin has a direct effect on proliferation of tumour cells in diabetic patients, the inhibitory effect of metformin on cancer progression may be indirect through decreased insulin concentrations. Insulin may favour cancer cell proliferation and mitogenic processes (Cohen \& LeRoith 2012). The gene encoding the insulin receptor (IR), by alternative splicing of exon 11, yields two isoforms: IR-A and IR-B. IR-A is principally overexpressed in cancer cells and differently from IR-B drives the mitogenic and proliferative pathways. IR-A and IR-B can form hybrid receptors activating the mitogenic pathway (Frasca et al. 2008). By binding to hybrid receptors, insulin stimulates specific IGF1 receptor (IGF1R) signalling pathways. In particular binding of insulin to insulin IR-A is the most accepted mechanism of mitogenic action of insulin. Insulin, even if with lower affinity, binds and activates IGF1R, which is expressed at high levels in tumour cells. Insulin decreases circulating concentrations of IGFBP1 and IGFBP2 (Jalving et al. 2010). This can improve the bioactivity of IGF1, which activates IGF1R and IR/IGF1R hybrid receptors with a similar potency (Slaaby et al. 2006). IR, IGF1R and IR/IGF1R phosphorylation activate the MAPK/ERK signalling pathway, stimulating cell growth and proliferation. Recently it has been demonstrated that MAPK/ERK can promote the Warburg effect, phosphorylating PKM2. PKM2 is one of the most important rate-limiting enzymes of glycolysis, which transfers phosphate from PEP to ADP generating ATP. MAPK/ERK induces Ser37 phosphorylation and nuclear translocation of PKM2. In the cytoplasm, PKM2 can exist as enzymatically active tetramer or as an inactive dimer. When a PKM2 dimer translocates

Published by Bioscientifica Ltd 
into the nucleus, it becomes a potent protein kinase which phosphorylates histone $\mathrm{H} 3$ leading to transcription of genes, including cyclin D1 and MYC (Yang et al. 2012). The alternative PI3K/AKT signalling pathway drives cellular protection and energy metabolism. Indeed, IR and IGF1R pathways may act on biochemical intermediates of glycolysis, and recently it has been proposed that PI3K/AKT signalling pathway may have a role in cellular energy metabolism. AKT is a master antiapoptotic regulator, which can favour glycolysis and glucose uptake, inducing the expression of hypoxia-inducible factor $1 \alpha$ and then of GLUT1 and HK2 in cancer cells (Semenza 2007). AKT phosphorylates HK2 at Thr473 and facilitates the association of HK2 with mitochondria, which is required not only to inhibit cytochrome $c$ release and apoptosis but also to ensure the catalytic function of HK2 (Roberts et al. 2014).

In this scenario, the question is whether and how metformin could interfere with the increased cellular energy disposition induced by insulin and IGF1 in cancer cells. In mouse models, oral administration of metformin inhibits the mTOR pathway in normal lung epithelium and lung tumours by decreasing insulin and IGF1 concentrations. Metformin inhibits IGF1-induced AKT phosphorylation (Salani et al. 2012). Thus, taking into account the existence of a cross-talk between AKT and mitochondria/HK2, this finding opens the possibility that metformin could drive dissociation of HK2 from mitochondria not only by a direct inhibitory effect but also by an indirect inhibitory effect (Salani et al. 2013; Fig. 3).

\section{Metformin's effect on glucose uptake: fluoro-deoxy-glucose imaging}

The signal transduction pathways that regulate the Warburg effect during tumourigenesis remain important points to be explored. For this reason, metformin's ability to inhibit glucose metabolism in cancer cells may offer a possible benefit by limiting energy resources and consequently affecting cancer cell proliferation and growth. One of the most relevant clinical uses of the Warburg effect relies on the fact that the high rate of deregulated glucose consumption can be visualised, non-invasively, for cancer diagnosis and staging. In fact, recent decades have witnessed an impressive growth of clinical application of positron emission tomography (PET) scanners due to their capability to map glucose consumption by the use of ${ }^{18}$ F-fluoro-deoxy-glucose (FDG). This analogue enters the cell via the same facilitative transporters of glucose; it is then phosphorylated to FDG6P and remains

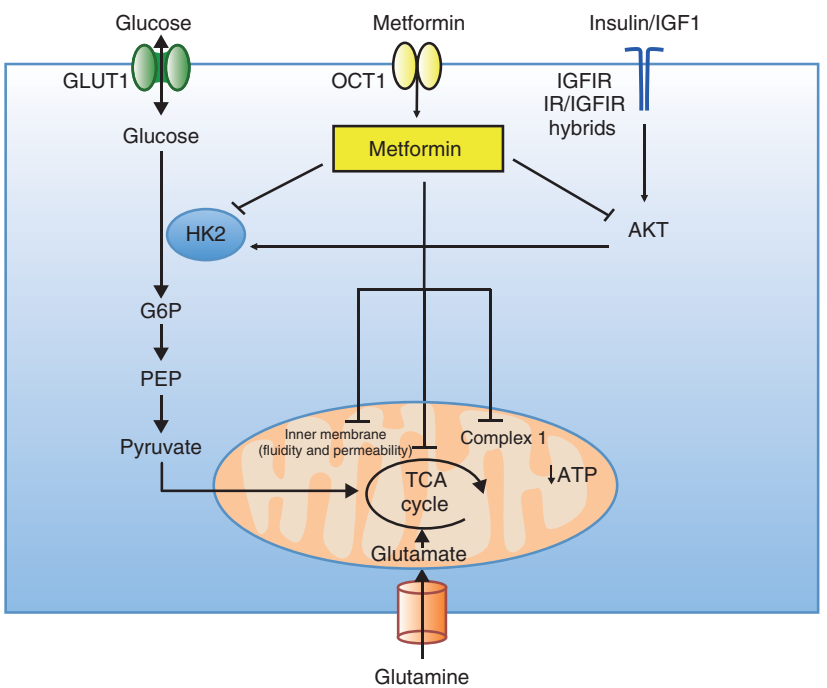

Figure 3

Proposed sites of action of metformin in cancer cells. The OCT1 transporter is highly expressed in cancer cells and mediates metformin uptake. Metformin's inhibitory action on glucose metabolism results from a combination of effects on glycolysis and growth factor signalling pathways. Metformin impairs glycolysis decreasing the enzymatic activity of the rate-limiting enzyme HK2. The inhibitory effect of metformin on AKT phosphorylation contributes to effects on HK2 by decreasing HK2 expression, activity and mitochondrial interaction. Metformin inhibits mitochondrial ATP production acting on respiratory chain complex 1 , decreasing TCA cycle intermediates and modifying the inner mitochondrial membrane.

trapped within the cytosol, being a false substrate for all further reactions channelling G6P to alternative pathways. The evaluation of metformin's effect in vitro on glucose uptake has been carried out in different cancer cellular models (Fang et al. 2012, Birsoy et al. 2014). As AMPK negatively regulates glucose consumption in cancer cells (Faubert et al. 2013), the effect of metformin on AMPK phosphorylation was associated with a significant doseand time-dependent reduction in FDG uptake. In a mouse model of prostate cancer, it has been shown that the decreased glucose oxidation exerted by metformin induces the dependency on reductive glutamine metabolism, which is an important source of cellular energy (Fendt et al. 2013). Glutamine is a substrate in the production of biochemical intermediates for the tricarboxylic acid (TCA) cycle. This anaplerotic function makes glutamine an important fuel and explains why several cancer cell lines consume glutamine at a higher rate than any other amino acid. Thus, interfering with glutamine metabolism may synergise with metformin's action in counteracting cancer growth and progression. Interestingly, in cancer stem cells (CSCs), metformin can deplete TCA cycle intermediates and alter the early metabolic state

Published by Bioscientifica Ltd. 
of the neoplastic transformation processes (Janzer et al. 2014). To overcome this apparent contradiction, it is worthy of note that CSCs have distinct phenotypes from the cells forming the bulk of the tumour and, in fact, are detectably more chemo- and radio-resistant than differentiated cancer cells. Despite few studies reporting on the metabolic effects of metformin on CSCs, encouraging results appear to indicate its effectiveness on CSCs viability. In particular, metformin seems to have an effect chemoresistant CDC subpopulations (Hirsch et al. 2009), acting preferentially on $\mathrm{CD}_{133^{+}}$(Gou et al. 2013) and CD $44^{\text {high }} / \mathrm{CD} 24^{\text {low }}$ cells (Zhu et al. 2014).

Owing to its peculiarity, the major advantage of FDG usage in clinical settings is linked with the short time needed to obtain a reliable estimate of glucose consumption. In fact, this technique allows early detection of the metabolic disturbance that is largely preceded by cell death. On one hand, this finding confirmed the robustness of the method, ruling out the possible contribution of cell loss to the low glucose consumption measured in cell cultures. On the other hand, it permitted elucidation the relevance of HK2 inhibition in cell ATP depletion, besides the well-known effect of metformin on mitochondrial respiratory complex 1. Also, usage of FDG confirmed the relevance of the glycolytic pathway in the maintenance of cancer growth and viability.

The extension of this model in vivo profoundly extended our understanding of the mechanism of action of metformin, documenting the role of indirect effects (caused by the modified whole-body metabolic patterns and signalling) and of direct actions on cancer glycolytic rate. The first evidence of metformin's effect in experimental cancer indicated that metformin can interfere with the diagnostic performance of FDG imaging in cancer detection and staging.

The tracer uptake in the different tissues is influenced by three main factors: i) lesion glucose consumption; ii) tracer availability in the blood; and iii) ratio between tracer and glucose plasma concentrations. Metformin markedly interferes with the latter two variables because its euglycemic action influences whole-body glucose disposal and thus FDG blood concentration. Moreover, this 'systemic tracer sequestration' is further amplified by the well-know increase in FDG uptake induced by metformin on colonic epithelium (Massollo et al. 2013), which further reduces tracer availability for cancer, independently from its own glucose consumption.

These considerations indicate that effect of metformin on cancer FDG uptake is related directly to the drug action on cancer metabolism and inversely to drug action on whole-body glucose disposal. The simple measurement of lesion tracer retention (the so-called SUV) might thus represent a suboptimal index of the responses of lesions to the drug. To overcome these limitations, the metabolic effect of metformin has been recently tested in a model of triple-negative breast cancer. In that study, compartmental analysis (Marini et al. 2013) of FDG exchange between blood and cancer revealed a significant reduction in cancer glucose consumption that was not paralleled by a corresponding decrease in lesion SUV (Figure S1, see section on supplementary data given at the end of this article). Altogether, these data thus confirmed that the inhibitory effect on tumour glucose consumption contributes to the anticancer potential of metformin.

\section{Drug development}

Biguanides (Fig. 1) offer a combination of unique chemical features allowing the possibility of easy ionization and tautomerization and thus constituting a privileged pharmacophore-interacting moiety with a drug-like scaffold suggestive of in vivo pharmacokinetics (Sundriyal et al. 2008). Among biguanides, metformin can be considered as a particular case because it is a small and highly hydrophilic molecule, which is not bound to plasma proteins, is readily filtered at the glomerulus and is poorly resorbed due to its low lipid solubility, overall resulting in high renal clearance. Thus, oral absorption, hepatic uptake and renal excretion of metformin are mediated by OTCs, reflecting the distribution, elimination and biochemical effects of metformin in tissues and subcellular compartments (Graham et al. 2011). As other biguanides have been already demonstrated to encompass a unique set of pharmacophore features conferring the ability to adapt to different biological targets, pharmacological effects can be conceivably diverse and adaptable to different tautomeric forms (Sundriyal et al. 2008). While this behaviour contributes to the puzzling polypharmacological role of metformin, several studies have recently contributed to uncovering mechanistic insights into key enzymes involved in the glycolytic pathway (Da Silva et al. 2010, Salani et al. 2013). For instance, as mentioned above, metformin is able to directly inhibit HK1 and HK2, and molecular modelling results support the idea of an allosteric inhibition, which raises the possibility of further chemical optimisations of the binding of the biguanide scaffold into the HK1/HK2 binding sites. Thus, biguanides can be considered as useful starting points for drug discovery purposes, embracing therapeutic indications going beyond the treatment of

Published by Bioscientifica Ltd 
type 2 diabetes, in particular, for cancer diseases (Martin \& Marais 2012). It should be noted that, while scientific data demonstrate the potential beneficial effects of metformin on several cancer types, a thorough assessment of the structure-activity relationship of other biguanides is yet to be demonstrated. This consideration is relevant to creation of a link with specific biological targets including HKs - and, in the long run, will be essential for embarking on new clinical trials based on the use of metformin or other biguanides (Suissa \& Azoulay 2012). It should be noted also that, because the expression of OCTs may differ between various tumour types, the direct action of metformin may be critically limited where expression of OTCs is low, while it could be greatly improved by conceiving new biguanide derivatives, or by repurposing known drugs such as phenformin or buformin. These drugs have been dismissed in the past due to the onset of severe lactic acidosis, but the mechanism underlying this side effect remains elusive. In addition, this risk might be not be contraindicated for dosing in cancer treatments, especially if the superior activity of these agents is clearly demonstrated (Sogame et al. 2013). Importantly, in the context of the development of new biguanide-based compounds, drug discovery techniques that include computer-aided molecular design tools such as molecular docking or pharmacophore screening, are expected to support the conception of new molecular entities with improved biological profiles (Del Rio et al. 2010, Caporuscio et al. 2011, Sanders et al. 2012).

\section{Perspectives}

Metformin is a first-line anti-diabetic drug showing interesting anticancer effects. In diabetic patients, it inhibits hepatic glucose production, by reducing cellular concentrations of ATP and then mimicking a fasting condition. To be responsive, metformin cells must express its facilitative transporter (OCT1). Several cancer cells express both OCT1 and HK2, making them responsive to the toxic action of metformin. In contrast, the prevalent expression of OCT1 and HK4, not inhibited by metformin, in the liver and the combined low OCT1 expression irrespective of the presence of HK2, in other tissues, allow its use in the prevention of massive cell death. In silico modelling has provide clues about this inhibitory mechanism opening avenues for investigating other HK2 inhibitors. These new compounds should still be dependent on OCT1 transport into cancer cells, preserving normal cells and representing an ideal anticancer treatment to kill cancer cells without affecting other tissues.

\section{Supplementary data}

This is linked to the online version of the paper at http://dx.doi.org/10.1530/ ERC-14-0284.

\section{Declaration of interest}

The authors declare that there is no conflict of interest that could be perceived as prejudicing the impartiality of this review.

\section{Funding}

This work was supported by MIUR (PRIN 2010JS3PMZ 009), the Italian Ministry of Health, Grant programme 2008 (G Sambuceti), the Italian Association for Cancer Research (AIRC Start Up grant number 6266 to A D Rio)

\section{References}

Alexander GC, Sehgal NL, Moloney RM \& Stafford RS 2008 National trends in treatment of type 2 diabetes mellitus, 1994-2007. Archives of Internal Medicine 168 2088-2094. (doi:10.1001/archinte.168.19.2088)

Aoki M, Terada T, Kajiwara M, Ogasawara K, Ikai I, Ogawa O, Katsura T \& Inui K 2008 Kidney-specific expression of human organic cation transporter 2 (OCT2/SLC22A2) is regulated by DNA methylation. American Journal of Physiology. Renal Physiology 295 F165-F170. (doi:10.1152/ajprenal.90257.2008)

Badrick E \& Renehan AG 2014 Diabetes and cancer: 5 years into the recent controversy. European Journal of Cancer $\mathbf{5 0}$ 2119-2125. (doi:10.1016/ j.ejca.2014.04.032)

Birsoy K, Possemato R, Lorbeer FK, Bayraktar EC, Thiru P, Yucel B, Wang T, Chen WW, Clish CB \& Sabatini DM 2014 Metabolic determinants of cancer cell sensitivity to glucose limitation and biguanides. Nature 508 108-112. (doi:10.1038/nature13110)

Caporuscio F, Rastelli G, Imbriano C \& Del Rio A 2011 Structure-based design of potent aromatase inhibitors by high-throughput docking. Journal of Medicinal Chemistry 54 4006-4017. (doi:10.1021/jm2000689)

Chen L, Pawlikowski B, Schlessinger A, More SS, Stryke D, Johns SJ, Portman MA, Chen E, Ferrin TE, Sali A et al. 2010 Role of organic cation transporter 3 (SLC22A3) and its missense variants in the pharmacologic action of metformin. Pharmacogenetics and Genomics 20 687-699. (doi:10.1097/FPC.0b013e32833fe789)

Choi M-K \& Song I-S 2008 Organic cation transporters and their pharmacokinetic and pharmacodynamic consequences. Drug Metabolism and Pharmacokinetics 23 243-253. (doi:10.2133/dmpk.23.243)

Cohen DH \& LeRoith D 2012 Obesity, type 2 diabetes, and cancer: the insulin and IGF connection. Endocrine-Related Cancer 19 F27-F45. (doi:10.1530/ERC-11-0374)

Da Silva D, Zancan P, Coelho WS, Gomez LS \& Sola-Penna M 2010 Metformin reverses hexokinase and 6-phosphofructo-1-kinase inhibition in skeletal muscle, liver and adipose tissues from streptozotocin-induced diabetic mouse. Archives of Biochemistry and Biophysics 496 53-60. (doi:10.1016/j.abb.2010.01.013)

Dowling RJ, Zakikhani M, Fantus IG, Pollak M \& Sonenberg N 2007 Metformin inhibits mammalian target of rapamycin-dependent translation initiation in breast cancer cells. Cancer Research $\mathbf{6 7}$ 10804-10812. (doi:10.1158/0008-5472.CAN-07-2310)

Drahota Z, Palenickova E, Endlicher R, Milerova M, Brejchova J, Vosahlikova M, Svoboda P, Kazdova L, Kalous M, Cervinkova Z et al. 2014 Biguanides inhibit complex I, II and IV of rat liver mitochondria and modify their functional properties. Physiological Research/Academia Scientiarum Bohemoslovaca 63 1-11. http://erc.endocrinology-journals.org DOI: 10.1530/ERC-14-0284
(C) 2014 Society for Endocrinology Printed in Great Britain 
Eechoute K, Sparreboom A, Burger H, Franke RM, Schiavon G, Verweij J, Loos WJ, Wiemer EA \& Mathijssen RH 2011 Drug transporters and imatinib treatment: implications for clinical practice. Clinical Cancer Research 17 406-415. (doi:10.1158/1078-0432.CCR-10-2250)

El-Mir MY, Nogueira V, Fontaine E, Avéret N, Rigoulet M \& Leverve X 2000 Dimethylbiguanide inhibits cell respiration via an indirect effect targeted on the respiratory chain complex I. Journal of Biological Chemistry 275 223-228. (doi:10.1074/jbc.275.1.223)

Evans JM, Donnelly LA, Emslie-Smith AM, Alessi DR \& Morris AD 2005 Metformin and reduced risk of cancer in diabetic patients. BMJ (Clinical research ed.) 330 1304-1305. (doi:10.1136/bmj.38415.708634.F7)

Fang R, Xiao T, Fang Z, Sun Y, Li F, Gao Y, Feng Y, Li L, Wang Y, Liu X et al. 2012 MicroRNA-143 (miR-143) regulates cancer glycolysis via targeting hexokinase 2 gene. Journal of Biological Chemistry 287 23227-23235. (doi:10.1074/jbc.M112.373084)

Faubert B, Boily G, Izreig S, Griss T, Samborska B, Dong Z, Dupuy F, Chambers C, Fuerth BJ, Viollet B et al. 2013 AMPK is a negative regulator of the Warburg effect and suppresses tumor growth in vivo. Cell Metabolism 17 113-124. (doi:10.1016/j.cmet.2012.12.001)

Fendt S-M, Bell EL, Keibler MA, Davidson SM, Wirth GJ, Fiske B, Mayers JR, Schwab M, Bellinger G, Csibi A et al. 2013 Metformin decreases glucose oxidation and increases the dependency of prostate cancer cells on reductive glutamine metabolism. Cancer Research 73 4429-4438. (doi:10.1158/0008-5472.CAN-13-0080)

Foretz M, Hébrard S, Leclerc J, Zarrinpashneh E, Soty M, Mithieux G, Sakamoto K, Andreelli F \& Viollet B 2010 Metformin inhibits hepatic gluconeogenesis in mice independently of the LKB1/AMPK pathway via a decrease in hepatic energy state. Journal of Clinical Investigation 120 2355-2369. (doi:10.1172/JCI40671)

Frasca F, Pandini G, Sciacca L, Pezzino V, Squatrito S, Belfiore A \& Vigneri R 2008 The role of insulin receptors and IGF-I receptors in cancer and other diseases. Archives of Physiology and Biochemistry 114 23-37. (doi:10.1080/13813450801969715)

Gou S, Cui P, Li X, Shi P, Liu T \& Wang C 2013 Low concentrations of metformin selectively inhibit $\mathrm{CD} 133^{+}$cell proliferation in pancreatic cancer and have anticancer action. PLOS ONE 8 e63969. (doi:10.1371/ journal.pone.0063969)

Graham GG, Punt J, Arora M, Day RO, Doogue MP, Duong JK, Furlong TJ, Greenfield JR, Greenup LC, Kirkpatrick CM et al. 2011 Clinical pharmacokinetics of metformin. Clinical Pharmacokinetics 50 81-98. (doi:10.2165/11534750-000000000-00000)

Grinfeld J, Gerrard G, Alikian M, Alonso-Dominguez J, Ale S, Valgañon M, Nteliopoulos G, White D, Marin D, Hedgley C et al. 2013 A common novel splice variant of SLC22A1 (OCT1) is associated with impaired responses to imatinib in patients with chronic myeloid leukaemia. British Journal of Haematology 163 631-639. (doi:10.1111/bjh.12591)

Gupta S, Wulf G, Henjakovic M, Koepsell H, Burckhardt G \& Hagos Y 2012 Human organic cation transporter 1 is expressed in lymphoma cells and increases susceptibility to irinotecan and paclitaxel. Journal of Pharmacology and Experimental Therapeutics 341 16-23. (doi:10.1124/ jpet.111.190561)

Hawley SA, Boudeau J, Reid JL, Mustard KJ, Udd L, Mäkelä TP, Alessi DR \& Hardie DG 2003 Complexes between the LKB1 tumor suppressor. STRAD $\alpha / \beta$ and MO25 $\alpha / \beta$ are upstream kinases in the AMP-activated protein kinase cascade. Journal of Biology 2 28. (doi:10.1186/ 1475-4924-2-28)

Hawley SA, Pan DA, Mustard KJ, Ross L, Bain J, Edelman AM, Frenguelli BG \& Hardie DG 2005 Calmodulin-dependent protein kinase kinase- $\beta$, is an alternative upstream kinase for AMP-activated protein kinase. Cell Metabolism 2 9-19. (doi:10.1016/j.cmet.2005.05.009)

Higgins JW, Bedwell DW \& Zamek-Gliszczynski MJ 2012 Ablation of both organic cation transporter (Oct)1 and Oct2 alters metformin pharmacokinetics but has no effect on tissue drug exposure and pharmacodynamics. Drug Metabolism and Disposition 40 1170-1177. (doi:10.1124/ dmd.112.044875)
Hirsch HA, Iliopoulos D, Tsichlis PN \& Struhl K 2009 Metformin selectively targets cancer stem cells, and acts together with chemotherapy to block tumor growth and prolong remission. Cancer Research 69 7507-7511. (doi:10.1158/0008-5472.CAN-09-2994)

Hundal HS, Ramlal T, Reyes R, Leiter LA \& Klip A 1992 Cellular mechanism of metformin action involves glucose transporter translocation from an intracellular pool to the plasma membrane in L6 muscle cells. Endocrinology 131 1165-1173. (doi:10.1210/en.131.3.1165)

Inoki K, Ouyang H, Zhu T, Lindvall C, Wang Y, Zhang X, Yang Q, Bennett C, Harada Y, Stankunas K et al. 2006 TSC2 Integrates Wnt and energy signals via a coordinated phosphorylation by AMPK and GSK3 to regulate cell growth. Cell 126 955-968. (doi:10.1016/j.cell.2006.06.055)

Iozzo P, Hallsten K, Oikonen V, Virtanen KA, Parkkola R, Kemppainen J, Solin O, Lonnqvist F, Ferrannini E, Knuuti J et al. 2003 Effects of metformin and rosiglitazone monotherapy on insulin-mediated hepatic glucose uptake and their relation to visceral fat in type 2 diabetes. Diabetes Care 26 2069-2074. (doi:10.2337/diacare.26.7.2069)

Jalving M, Gietema JA, Lefrandt JD, De Jong S, Reyners AK, Gans RO \& De Vries EG 2010 Metformin: taking away the candy for cancer? European Journal of Cancer 46 2369-2380. (doi:10.1016/j.ejca.2010. 06.012)

Janzer A, German NJ, Gonzalez-Herrera KN, Asara JM, Haigis MC \& Struhl K 2014 Metformin and phenformin deplete tricarboxylic acid cycle and glycolytic intermediates during cell transformation and NTPs in cancer stem cells. PNAS 111 10574-10579. (doi:10.1073/pnas.1409844111)

Komori T, Hotta N, Kobayashi M, Sakakibara F, Koh N \& Sakamoto N 1993 Biguanides may produce hypoglycemic action in isolated rat hepatocytes through their effects on L-alanine transport. Diabetes Research and Clinical Practice 22 11-17. (doi:10.1016/0168-8227(93)90127-Q)

Koppenol WH, Bounds PL \& Dang CV 2011 Otto Warburg's contributions to current concepts of cancer metabolism. Nature Reviews. Cancer 11 325-337. (doi:10.1038/nrc3038)

Koren-Michowitz M, Buzaglo Z, Ribakovsky E, Schwarz M, Pessach I, Shimoni A, Beider K, Amariglio N, le-Coutre P \& Nagler A 2013 OCT1 genetic variants are associated with long term outcomes in imatinib treated chronic myeloid leukemia patients. European Journal of Haematology 92 283-288. (doi:10.1111/ejh.12235)

Kristensen JM, Treebak JT, Schjerling P, Goodyear L \& Wojtaszewski JF 2014 Two weeks of metformin treatment induces AMPK-dependent enhancement of insulin-stimulated glucose uptake in mouse soleus muscle. American Journal of Physiology. Endocrinology and Metabolism 306 E1099-E1109. (doi:10.1152/ajpendo.00417.2013)

Li S, Chen Y, Zhang S, More SS, Huang X \& Giacomini KM 2011 Role of organic cation transporter 1 , OCT1 in the pharmacokinetics and toxicity of cis-diammine(pyridine)chloroplatinum(II) and oxaliplatin in mice. Pharmaceutical Research 28 610-625. (doi:10.1007/s11095010-0312-6)

Madiraju AK, Erion DM, Rahimi Y, Zhang X-M, Braddock DT, Albright RA, Prigaro BJ, Wood JL, Bhanot S, MacDonald MJ et al. 2014 Metformin suppresses gluconeogenesis by inhibiting mitochondrial glycerophosphate dehydrogenase. Nature 26 542-546. (doi:10.1038/nature13270)

Marini C, Salani B, Massollo M, Amaro A, Esposito AI, Orengo AM, Capitanio S, Emionite L, Riondato M, Bottoni G et al. 2013 Direct inhibition of hexokinase activity by metformin at least partially impairs glucose metabolism and tumor growth in experimental breast cancer. Cell Cycle 12 3490-3499. (doi:10.4161/cc.26461)

Martin M \& Marais R 2012 Metformin: a diabetes drug for cancer, or a cancer drug for diabetics? Journal of Clinical Oncology 30 2698-2700. (doi:10.1200/JCO.2012.42.1677)

Massollo M, Marini C, Brignone M, Emionite L, Salani B, Riondato M, Capitanio S, Fiz F, Democrito A, Amaro A et al. 2013 Metformin temporal and localized effects on gut glucose metabolism assessed using ${ }^{18}$ F-FDG PET in mice. Journal of Nuclear Medicine $\mathbf{5 4} 259-266$ (doi:10.2967/jnumed.112.106666)

Memmott RM, Mercado JR, Maier CR, Kawabata S, Fox SD \& Dennis PA 2010 Metformin prevents tobacco carcinogen-induced lung 
tumorigenesis. Cancer Prevention Research 3 1066-1076. (doi:10.1158/ 1940-6207.CAPR-10-0055)

Miller RA, Chu Q, Xie J, Foretz M, Viollet B \& Birnbaum MJ 2013 Biguanides suppress hepatic glucagon signalling by decreasing production of cyclic AMP. Nature 494 256-260. (doi:10.1038/nature11808)

Natali A \& Ferrannini E 2006 Effects of metformin and thiazolidinediones on suppression of hepatic glucose production and stimulation of glucose uptake in type 2 diabetes: a systematic review. Diabetologia 49 434-441. (doi:10.1007/s00125-006-0141-7)

Nies AT, Koepsell H, Winter S, Burk O, Klein K, Kerb R, Zanger UM, Keppler D, Schwab M \& Schaeffeler E 2009 Expression of organic cation transporters OCT1 (SLC22A1) and OCT3 (SLC22A3) is affected by genetic factors and cholestasis in human liver. Hepatology $\mathbf{5 0}$ 1227-1240. (doi:10.1002/hep.23103)

Nies AT, Schaeffeler E, van der Kuip H, Cascorbi I, Bruhn O, Kneba M, Pott C, Hofmann U, Volk C, Hu S et al. 2014 Cellular uptake of imatinib into leukemic cells is independent of human organic cation transporter 1 (OCT1). Clinical Cancer Research 1 985-994. (doi:10.1158/1078-0432.CCR-13-1999)

Omar H, Berman-Booty L, Kulp SK \& Chen C-S 2010 Energy restriction as an antitumor target. Future Oncology 6 1675-1679. (doi:10.2217/ fon.10.130)

Owen MR, Doran E \& Halestrap AP 2000 Evidence that metformin exerts its anti-diabetic effects through inhibition of complex 1 of the mitochondrial respiratory chain. Biochemical Journal 348 607-614. (doi:10.1042/0264-6021:3480607)

Pastorino JG \& Hoek JB 2003 Hexokinase II: the integration of energy metabolism and control of apoptosis. Current Medicinal Chemistry 10 1535-1551. (doi:10.2174/0929867033457269)

Patra K, Wang Q, Bhaskar P, Miller L, Wang Z, Wheaton W, Chandel N, Laakso M, Muller W, Allen E et al. 2013 Hexokinase 2 is required for tumor initiation and maintenance and its systemic deletion is therapeutic in mouse models of cancer. Cancer Cell 24 213-228. (doi:10.1016/j.ccr.2013.06.014)

Pollak M 2012 The insulin and insulin-like growth factor receptor family in neoplasia: an update. Nature Reviews. Cancer 12 159-169. (doi:10.1038/nrc3215)

Radziuk J, Zhang Z, Wiernsperger N \& Pye S 1997 Effects of metformin on lactate uptake and gluconeogenesis in the perfused rat liver. Diabetes 46 1406-1413. (doi:10.2337/diab.46.9.1406)

Del Rio A, Barbosa AJ, Caporuscio F \& Mangiatordi GF 2010 CoCoCo: a free suite of multiconformational chemical databases for highthroughput virtual screening purposes. Molecular BioSystems 6 2122-2128. (doi:10.1039/c0mb00039f)

Roberts DJ, Tan-Sah VP \& Smith JM 2014 Akt phosphorylates HK-II at Thr-473 and increases mitochondrial HK-II association to protect cardiomyocytes. Journal of Biological Chemistry 288 23798-23806. (doi:10.1074/jbc.M113.482026)

Sajan MP, Bandyopadhyay G, Miura A, Standaert ML, Nimal S, Longnus SL, Van Obberghen E, Hainault I, Foufelle F, Kahn R et al. 2010 AICAR and metformin, but not exercise, increase muscle glucose transport through AMPK-, ERK-, and PDK1-dependent activation of atypical PKC. American Journal of Physiology. Endocrinology and Metabolism 298 E179-E192. (doi:10.1152/ajpendo.00392.2009)

Salani B, Maffioli S, Hamoudane M, Parodi A, Ravera S, Passalacqua M, Alama A, Nhiri M, Cordera R \& Maggi D 2012 Caveolin-1 is essential for metformin inhibitory effect on IGF1 action in non-small-cell lung cancer cells. FASEB Journal 26 788-798. (doi:10.1096/fj.11192088)

Salani B, Marini C, Del Rio A, Ravera S, Massollo M, Orengo AM, Amaro A, Passalacqua M, Maffioli S, Pfeffer U et al. 2013 Metformin impairs glucose consumption and survival in Calu-1 cells by direct inhibition of hexokinase-II. Scientific Reports 3 2070. (doi:10.1038/srep02070)

Sanders MP, Barbosa AJ, Zarzycka B, Nicolaes GA, Klomp JP, de Vlieg J \& Del Rio A 2012 Comparative analysis of pharmacophore screening tools. Journal of Chemical Information and Modeling 52 1607-1620. (doi:10.1021/ci2005274)

Segal ED, Yasmeen A, Beauchamp M-C, Rosenblatt J, Pollak M \& Gotlieb WH 2011 Relevance of the OCT1 transporter to the antineoplastic effect of biguanides. Biochemical and Biophysical Research Communications 414 694-699. (doi:10.1016/j.bbrc.2011.09.134)

Semenza GL 2007 Hypoxia-inducible factor 1 (HIF-1) pathway. Science Signaling 2007 cm8. (doi:10.1126/stke.4072007cm8)

Shaw RJ, Kosmatka M, Bardeesy N, Hurley RL, Witters LA, DePinho RA \& Cantley LC 2004 The tumor suppressor LKB1 kinase directly activates AMP-activated kinase and regulates apoptosis in response to energy stress. PNAS 101 3329-3335. (doi:10.1073/pnas.0308061100)

Shaw RJ, Lamia KA, Vasquez D, Koo S-H, Bardeesy N, Depinho RA, Montminy M \& Cantley LC 2005 The kinase LKB1 mediates glucose homeostasis in liver and therapeutic effects of metformin. Science $\mathbf{3 1 0}$ 1642-1646. (doi:10.1126/science.1120781)

Shu Y, Brown C, Castro RA, Shi RJ, Lin ET, Owen RP, Sheardown SA, Yue L, Burchard EG, Brett CM et al. 2008 Effect of genetic variation in the organic cation transporter 1, OCT1, on metformin pharmacokinetics. Clinical Pharmacology and Therapeutics 83 273-280. (doi:10.1038/sj.clpt. 6100275)

Slaaby R, Schäffer L, Lautrup-Larsen I, Andersen AS, Shaw AC, Mathiasen IS \& Brandt J 2006 Hybrid receptors formed by insulin receptor (IR) and insulin-like growth factor I receptor (IGFIR) have low insulin and high IGF-1 affinity irrespective of the IR splice variant. Journal of Biological Chemistry 281 25869-25874. (doi:10.1074/jbc.M605189200)

Sogame Y, Kitamura A, Yabuki M, Komuro S \& Takano M 2013 Transport of biguanides by human organic cation transporter OCT2. Biomedicine \& Pharmacotherapy 67 425-430. (doi:10.1016/j.biopha.2013. 02.003)

Stevens RJ, Ali R, Bankhead CR, Bethel MA, Cairns BJ, Camisasca RP, Crowe FL, Farmer AJ, Harrison S, Hirst JA et al. 2012 Cancer outcomes and all-cause mortality in adults allocated to metformin: systematic review and collaborative meta-analysis of randomised clinical trials. Diabetologia 55 2593-2603. (doi:10.1007/s00125-012-2653-7)

Suissa S \& Azoulay L 2012 Metformin and the risk of cancer: time-related biases in observational studies. Diabetes Care 35 2665-2673. (doi:10.2337/dc12-0788)

Sundriyal S, Khanna S, Saha R \& Bharatam PV 2008 Metformin and glitazones: does similarity in biomolecular mechanism originate from tautomerism in these drugs? Journal of Physical Organic Chemistry 21 30-33. (doi:10.1002/poc.1273)

Takane H, Shikata E, Otsubo K, Higuchi S \& Ieiri I 2008 Polymorphism in human organic cation transporters and metformin action. Pharmacogenomics 9 415-422. (doi:10.2217/14622416.9.4.415)

Vander Heiden MG, Cantley LC \& Thompson CB 2009 Understanding the Warburg effect: the metabolic requirements of cell proliferation. Science 324 1029-1033. (doi:10.1126/science.1160809)

Viollet B, Guigas B, Sanz Garcia N, Leclerc J, Foretz M \& Andreelli F 2011 Cellular and molecular mechanisms of metformin: an overview. Clinical Science 122 253-270. (doi:10.1042/CS20110386)

Wahdan-Alaswad R, Fan Z, Edgerton SM, Liu B, Deng X-S, Arnadottir SS Richer JK, Anderson SM \& Thor AD 2013 Glucose promotes breast cancer aggression and reduces metformin efficacy. Cell Cycle $\mathbf{1 2}$ 3759-3769. (doi:10.4161/cc.26641)

Watson J 2014 Type 2 diabetes as a redox disease. Lancet 383 841-843. (doi:10.1016/S0140-6736(13)62365-X)

Wilson JE 2003 Isozymes of mammalian hexokinase: structure, subcellular localization and metabolic function. Journal of Experimental Biology 206 2049-2057. (doi:10.1242/jeb.00241)

Wollen N \& Bailey CJ 1988 Inhibition of hepatic gluconeogenesis by metformin. Synergism with insulin. Biochemical Pharmacology $\mathbf{3 7}$ 4353-4358. (doi:10.1016/0006-2952(88)90617-X)

Yang W, Zheng Y, Xia Y, Ji H, Chen X, Guo F, Lyssiotis CA, Aldape K, Cantley LC \& Lu Z 2012 ERK1/2-dependent phosphorylation and 
nuclear translocation of PKM2 promotes the Warburg effect. Nature Cell Biology 14 1295-1304. (doi:10.1038/ncb2629)

Zakikhani M, Dowling R, Fantus IG, Sonenberg N \& Pollak M 2006 Metformin is an AMP kinase-dependent growth inhibitor for breast cancer cells. Cancer Research 66 10269-10273. (doi:10.1158/0008-5472. CAN-06-1500)

Zhang L, Dresser MJ, Gray AT, Yost SC, Terashita S \& Giacomini KM 1997 Cloning and functional expression of a human liver organic cation transporter. Molecular Pharmacology 51 913-921. (doi:10.1124/mol.51. 6.913)

Zhang S, Lovejoy KS, Shima JE, Lagpacan LL, Shu Y, Lapuk A, Chen Y, Komori T, Gray JW, Chen X et al. 2006 Organic cation transporters are determinants of oxaliplatin cytotoxicity. Cancer Research 66 8847-8857. (doi:10.1158/0008-5472.CAN-06-0769)
Zhang P, Li H, Tan X, Chen L \& Wang S 2013 Association of metformin use with cancer incidence and mortality: a meta-analysis. Cancer Epidemiology 37 207-218. (doi:10.1016/j.canep.2012.12.009)

Zhu P, Davis M, Blackwelder AJ, Bachman N, Liu B, Edgerton S, Williams LL, Thor AD \& Yang X 2014 Metformin selectively targets tumor-initiating cells in ErbB2-overexpressing breast cancer models. Cancer Prevention Research 7 199-210. (doi:10.1158/1940-6207. CAPR-13-0181)

Zordoky BN, Bark D, Soltys CL, Sung MM \& Dyck JR 2014 The anti-proliferative effect of metformin in triple-negative MDA-MB-231 breast cancer cells is highly dependent on glucose concentration: implications for cancer therapy and prevention. Biochimica et Biophysica Acta 1840 1943-1957. (doi:10.1016/j.bbagen. 2014.01.023)

Received in final form 26 September 2014

Accepted 1 October 2014

Made available online as an Accepted Preprint

1 October 2014
Published by Bioscientifica Ltd. 